\title{
Endoscopic retrograde cholangiopancreatography (ERCP)-related esophageal perforation in osteo- genesis imperfecta
}

A 77-year-old woman with osteogenesis imperfecta was admitted with symptomatic choledocholithiasis. Endoscopic retrograde cholangiopancreatography (ERCP) was carried out and mild resistance was encountered in the distal esophagus with "red out" during intubation. The duodenoscope was withdrawn and gastroscopy revealed a tear in the distal esophagus ( $\bullet$ Fig. 1). As a perforation was suspected, a nasogastric tube was deployed, intravenous antibiotics were started, and surgery was consulted. An esophagogram confirmed the esophageal leak ( Fig.2) and thoraco-abdominal computed tomography (CT) showed a $0.7-\mathrm{cm}$ esophageal perforation and airfluid collection $(3 \mathrm{~cm} \times 10 \mathrm{~cm})$ in the posterior mediastinum ( $\bullet$ Fig.3). Conservative management was decided on due to clinical stability and poor operative candidacy. Within 48 hours the liver function tests normalized and parenteral nutrition was started. The day 7 esophagogram showed partial healing. Enteral feeding was started with a nasojejunal tube placed fluoroscopically. An esophagogram on day 27 showed complete healing ( Fig.4). The patient started an oral diet and was discharged with plan for elective cholecystectomy.

Osteogenesis imperfecta is an autosomal dominant disorder of collagen synthesis
( 1 in 10000 births). Gastrointestinal manifestations in osteogenesis imperfecta are rare although spontaneous colon perforation has been reported, where histopathological examination showed absence of muscle layers in the perforated segment [1]. Murine models of osteogenesis imperfecta showed thinner esophageal submucosa and muscularis compared with wild mice [2]. ERCP-related perforations are infrequent $(0.4 \%)$ and are commonly related to improper guidewire insertion or sphincterotomy [3]. Esophageal perforation in ERCP is rare and is attributed to use of sharp accessories $[3,4]$. This is the first reported case of esophageal perforation in osteogenesis imperfecta. We believe that perforation in our patient occurred due to abnormal esophageal submucosa and muscularis and limited view with duodenoscope. Esophageal perforations are traditionally managed surgically; however, good outcomes are reported with conservative management in smaller perforations and contained leaks [5]. The present case highlights the propensity for esophageal perforation in osteogenesis imperfecta. We recommend caution and low threshold to suspect perforation during gastrointestinal endoscopy in osteogenesis imperfecta.
Endoscopy_UCTN_Code_CPL_1AK_2AC

Competing interests: None

\section{R. Kochar ${ }^{1}$, R. Pandey², W. Mileski ${ }^{3}$, S. D. Lick ${ }^{3}$, M. H. Schreiber ${ }^{4}$, S. V. J. Parupudi ${ }^{1}$}

${ }^{1}$ Division of Gastroenterology and Hepatology, The University of Texas Medical Branch, Galveston, Texas, USA

2 University of Arkansas for Medical Sciences, Little Rock, Arkansas, USA

${ }^{3}$ Department of Surgery, The University of Texas Medical Branch, Galveston, Texas, USA

${ }^{4}$ Department of Radiology, The University of Texas Medical Branch, Galveston, Texas, USA

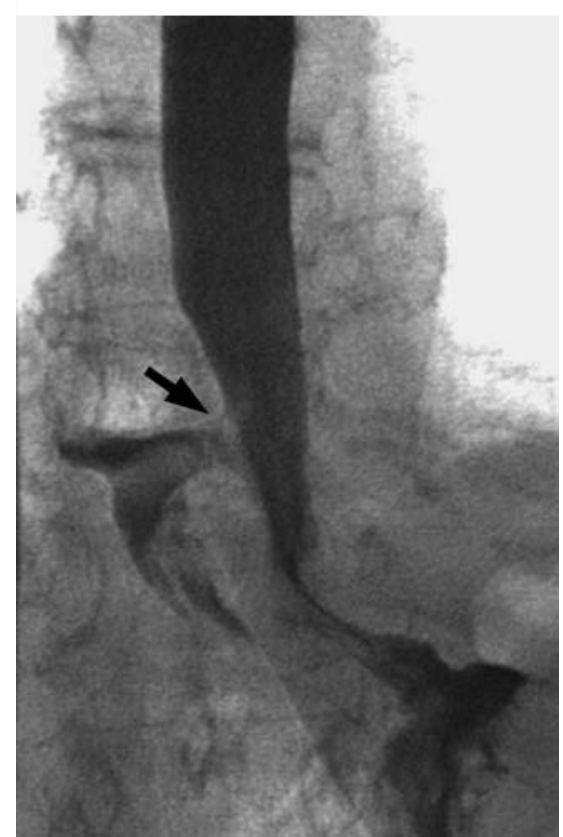

Fig.2 Esophagogram with water-soluble contrast demonstrating the distal esophageal leak (black arrow). 


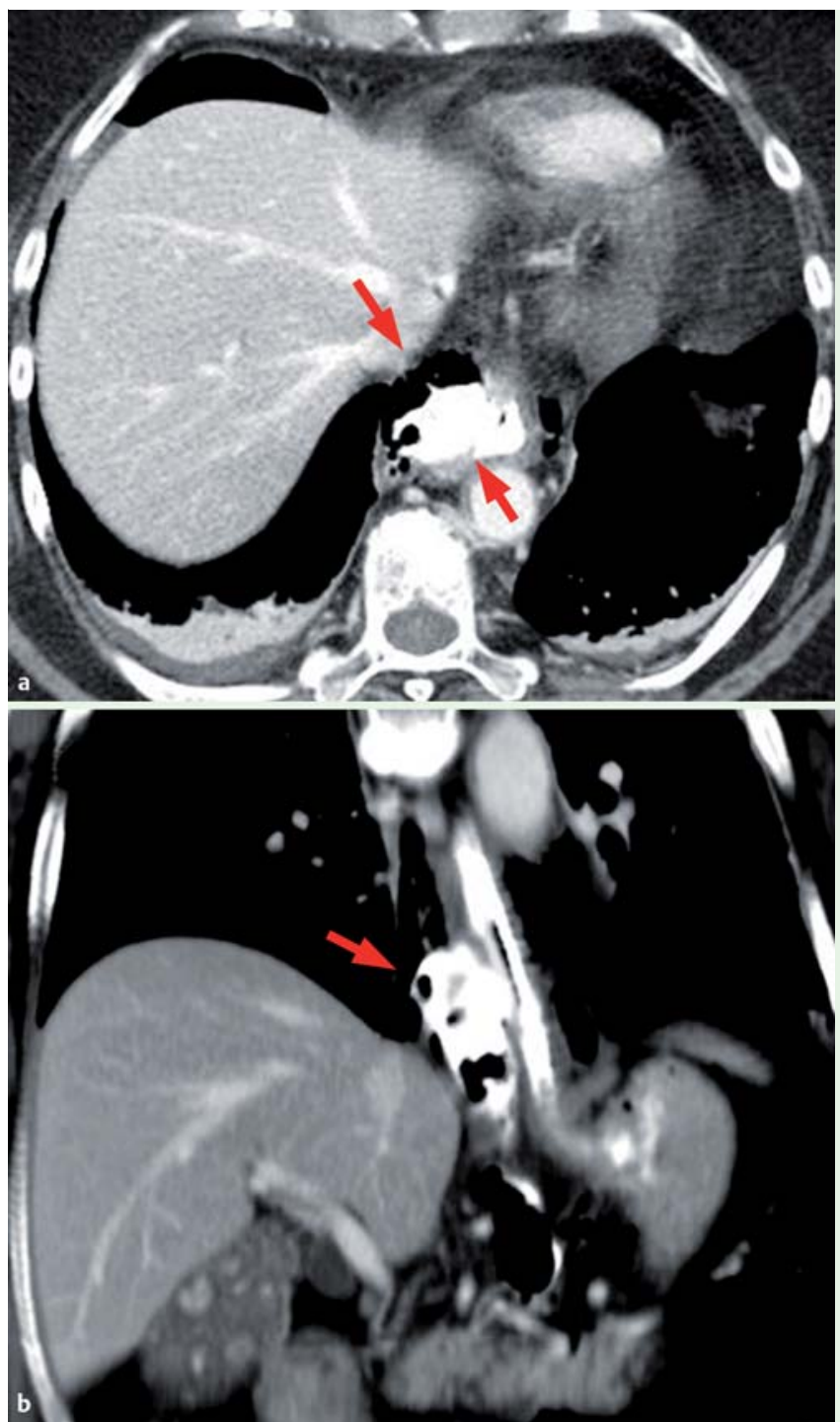

Fig. 3 Thoraco-abdominal computed tomography (CT) scan showing paraesophageal pooling of the oral contrast (arrows on transverse section, a) the retroperitoneum (arrow on coronal section, $\mathbf{b})$.

\section{References}

1 Wheatley K, Heng EL, Sheppard M et al. A case of spontaneous intestinal perforation in osteogenesis imperfecta. J Clin Med Res 2010; 2 (Suppl. 04): 198-200

2 Gregersen $H$, Weis SM, McCulloch AD. Oesophageal morphometry and residual strain in a mouse model of osteogenesis imperfecta. Neurogastroenterol Motil 2001; 13 (Suppl. 05): 457-464 tracking down towards

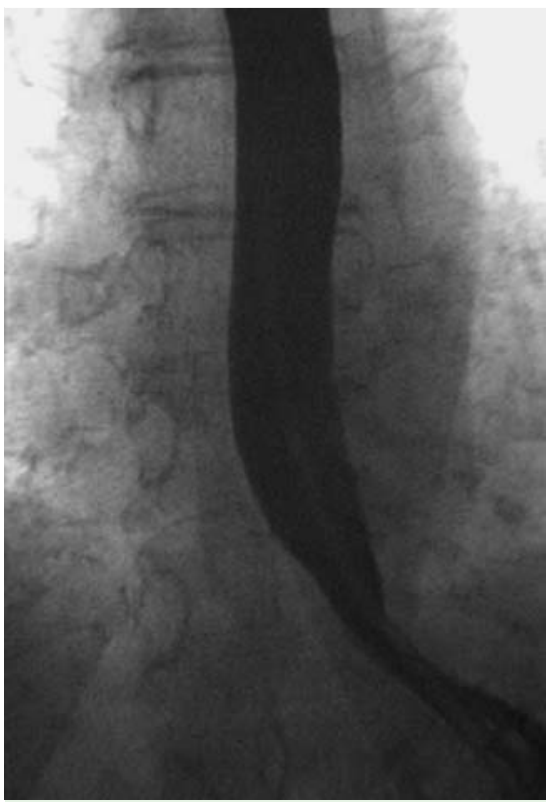

Fig.4 Esophagogram with water-soluble contrast on day 27 showing complete healing.

Bibliography

DOI http://dx.doi.org/

10.1055/s-0032-1308930

Endoscopy 2012; 44: E238-E239

(c) Georg Thieme Verlag KG

Stuttgart · New York

ISSN 0013-726X

\section{Corresponding author \\ Rajan Kochar}

Division of Gastroenterology and Hepatology The University of Texas Medical Branch 301 University Boulevard RT 0764 Galveston

Texas 77555

USA

3 Enns R, Eloubeidi MA, Mergener $K$ et al. ERCP related perforations - risk factors and management. Endoscopy 2002; 34: 293-298

4 Fireman Z, Kyzer S, Michalevicz D et al. Esophageal perforation after endoscopic sphincterotomy during stone extraction from the common bile duct. J Clin Gastroenterol 1994; 19 (Suppl. 02): 173 - 175

5 Sepesi B, Raymond DP, Peters JH. Esophageal perforation: surgical, endoscopic and medical management strategies. Curr Opin Gastroenterol 2010; 26 (Suppl. 04): 379-383 